\title{
CONTROLE ADAPTATIVO USADO EM DOIS ELOS DE UM ROBÔ ELETROMECÂNICO DE CINCO GRAUS DE LIBERDADE
}

\author{
José Antônio Riul ${ }^{(1)}$ (riul@ct.ufpb.br), Paulo Henrique de Miranda Montenegro ${ }^{(2)}$ (paulo@ct.ufpb.br) \\ Naor Moraes Melo ${ }^{(3)}$ (naor@ct.ufpb.br) \\ (1) Universidade Federal da Paraíba (UFPB); Departamento de Engenharia Mecânica \\ (2) ) Universidade Federal da Paraíba (UFPB); Departamento de Engenharia Mecânica \\ (3) Universidade Federal da Paraíba (UFPB); Departamento de Engenharia Mecânica
}

RESUMO. Este trabalho tem como objetivo o projeto de controladores adaptativos para controle de posição de dois elos de um robo eletromecânico de cinco graus de liberdade (5 GDL). O controle é realizado através de simulação, usando modelos dos elos obtidos com dados reais do sistema, através de estimação de parâmetros usando o algoritmo dos mínimos quadrados recursivo (MQR). A identificação dos elos é realizada em malha aberta, utilizando dados reais coletados do robo. São projetados e implementados controladores adaptativos via imposição de pólos para o controle de posição dos dois elos. Os controladores são projetados em malha fechada com base nos modelos obtidos para os elos. A estratégia de controle a ser usada baseia-se em um modelo explícito do sistema. Os controladores são implementados através de um programa computacional. São mostrados como resultados as curvas de saida dos modelos dos dois elos, em malha aberta e as curvas de saída dos elos sob ação dos controladores adaptativos, em malha fechada.

PALAVRAS-CHAVE: Robótica, Identificação de sistemas, Controle Adaptativo

\section{ADAPTIVE CONTROL USED IN TWO LINKS OF A ELECTROMECHANICAL ROBOT OF- FIVE DEGREE FREEDOM}

ABSTRACT: This work has as objective the design of adaptive controllers to control the position of two links of an electromechanical robot of five degrees of freedom (5 GDL). The control is performed through simulation, using models of the links obtained with real system data, through parameter estimation using the least squares recursive algorithm (MQR). The identification of the links is done in open loop, using real data collected from the robot. Adaptive controllers are designed and implemented via the imposition of poles to control the position of the two links. The controllers are designed in closed loop based on the models obtained for the links. The control strategy to be used is based on an explicit model of the system. The controllers are implemented through a computer program. The output curves of the two open loop models are shown as results and the output curves of the links under the action of adaptive controllers, in closed loop.

KEYWORDS: Robotics, Systems, Identification, Adaptive Control 


\section{XIX CENEMI}

\section{INTRODUÇÃO}

O presente trabalho tem como objetivo o modelamento linear de dois elos de um robô manipulador eletromecânico de cinco graus de liberdade (5 GDL) e o projeto de controladores adaptativos para os dois elos. Os elos 1 e 2 a serem controlados são mostrados na Figura 1 . 0 modelo matemático de um sistema pode ser obtido através de leis físicas, conhecido como modelo caixa branca ou por técnica de identificação paramétrica, conhecida como modelo caixa preta, que depende de dados reais do sistema.

Modelos caixa branca de robôs manipuladores são não lineares (SPONG e VIDYASAGAR, 1989), (CRAIG, 1989), enquanto que a identificação caixa preta, gera modelos lineares e não lineares (AGUIRRE, 2000), que podem ser usados para projeto e implementação de controladores adaptativos. Os modelos caixa branca, quando utilizados em projetos de controladores, exigem uma quantidade elevada de cálculos, o que torna necessário o uso de máquinas de grande porte, tendo em vista o esforço computacional requerido (KOIVO e GUO, 1983), (SHIH e TSENG, 1995).

$\mathrm{Na}$ utilização de modelos caixa preta, suas estruturas são definidas a priori, e com isto, a escolha de modelos de primeira ou segunda ordem, que representam bem os sistemas reais, e que requerem baixo esforço computacional, são empregados.

As ações de controle adaptativo serão realizadas por dois controladores auto-ajustáveis; via imposição de pólos. Os controladores da família PID constituem cerca de 90\% dos instalados atualmente na indústria (BEHAR e IRANZO, 2003). Como resultados deste trabalho, são apresentadas curvas das respostas estimadas, obtidas com o modelamento dos dois elos do robo e curvas das respostas dos elos sobre ação dos controladores adaptativos projetados, obtidas por simulação.

\section{DESCRIÇÃO DO SISTEMA}

O Robô Manipulador, mostrado na Figura 1 é um robô didático, pesando aproximadamente 7 kg, referência RD5NT, fabricado pela empresa Didacta Itália, é composto de cinco juntas rotativas, quatro elos e uma garra. A primeira junta rotativa refere-se ao movimento angular da base, com deslocamento máximo de 293으, a segunda junta rotativa refere-se ao ombro, com deslocamento angular máximo de $107^{\circ}$, a terceira junta rotativa refere-se ao cotovelo, com deslocamento angular máximo de $284^{\circ}$, a quarta junta rotativa refere-se ao pulso com deslocamento angular máximo de $360^{\circ}$ e a quinta junta rotativa refere-se a um sistema corôa / parafuso sem fim, responsável pelo curso da garra, de no máximo $22 \mathrm{~mm}$, capacidade de fixação de carga de 350 gramas e parada automática por meio de uma micro chave operando com velocidade de fechamento ajustável. Os elos do robô manipulador representam o tronco, o braço, o antebraço e o punho. A transmissão de cada movimento é feita por meio de bloco motor redutor, com dois estágios de redução, e com relação de transmissão total de 1/500. Os motores dos blocos são de corrente continua, referência 2139.906-22.112-050, fabricados pela Maxon Motor, com potencia de 2,5 watts e com capacitor de longa vida. A voltagem nominal dos motores CC é de 12 volts e a rotação máxima sem carga é de $6480 \mathrm{rpm}$. A medição dos deslocamentos angulares das juntas e movimento da garra é assegurada por meio de potenciômetros rotativos lineares, referência 78CSB502, fabricados pela Sfernice, com resistência de $5 \mathrm{k} \Omega$. 


\section{XIX \\ CONEMI}

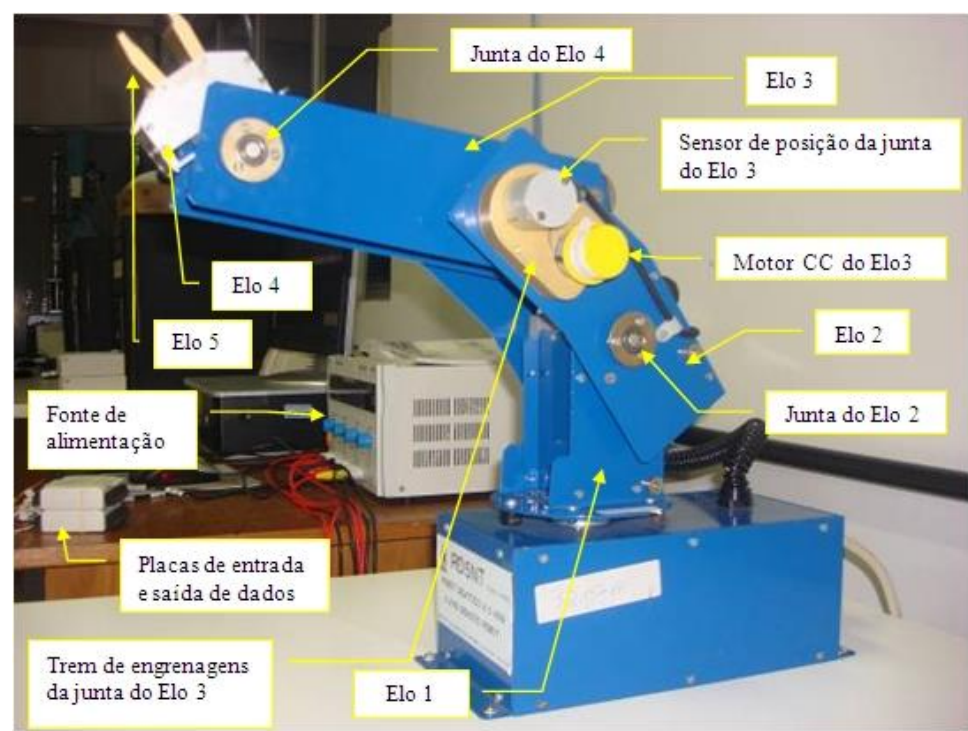

Figura 1. Robô manipulador de cinco gdl

Um computador HP Compaq com processador AMD Athlon dual core de 985 Mhz e 786 MB de RAM é utilizado para enviar comando de acionamento aos motores $\mathrm{CC}$ e receber os sinais dos sensores potenciométricos. A comunicação do robô com o computador é realizada através de duas placas de entrada e saída de dados, NI USB-6009 e de um programa computacional nas plataformas LabView e Matlab. Considerando as características de tensão e capacidade máxima de corrente das placas de entrada e saída de dados, houve a necessidade de introdução de um amplificador de potência para servir como fonte de suprimento aos motores de corrente contínua do robô manipulador. Este amplificador além de fornecer a potência necessária ao acionamento de cada motor, fornece a polaridade adequada para que o seu funcionamento se dê no sentido desejado. A decisão do sentido de rotação depende da tensão de excitação aplicada em seus terminais de entrada.

\section{CONTROLADOR AUTO-AJUSTÁVEL (STR - Self-Tuning Regulator)}

O controlador auto-ajustável (STR) é um tipo de controlador adaptativo, que automatiza as tarefas de modelagem matemática, projeto e implementação da lei de controle. O STR é explícito quando as tarefas de modelagem matemática, projeto e implementação da lei de controle são realizadas a partir dos parâmetros estimados da planta. No STR, os parâmetros estimados do sistema são determinados e atualizados, à cada período de amostragem, através do MQR (Minimos Quadrados Recursivos). O diagrama de blocos de um STR explícito é apresentado na Figura 2. No diagrama de blocos destacam-se duas malhas fechadas. A malha inferior composta pelo sistema e pela realimentação de saída, e a superior composta pelo estimador de parâmetros, pelo projeto da lei de controle e pelo controlador ajustável. No STR os parâmetros estimados são considerados como se fossem os parâmetros reais do sistema. Esta abordagem é baseada no princípio da equivalência à certeza, conforme HEMERLY (1996). 


\section{$\mathrm{XIX}$ \\ CENEMI}

Controladores como: Proporcional Integral Derivativo (PID), Proporcional Integral (PI), Proporcional Derivativo (PD), de Variância Mínima (MV), de Variância Mínima Generalizado (GMV), Linear Quadrático Gaussiano (LQG) e Controlador Preditivo Generalizado (GPC), podem ser utilizados para o projeto da lei de controle.

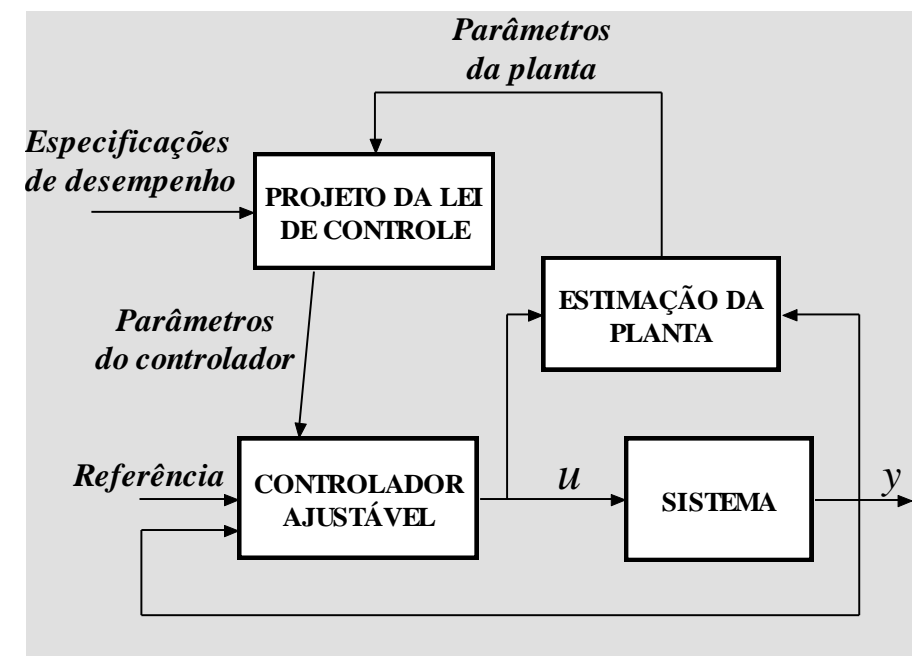

Figura 2. Diagrama de blocos do STR explícito

A estimação dos parâmetros do sistema é a essência do STR. Dentre os algoritmos aplicáveis à esta tarefa cita-se o dos mínimos quadrados recursivos (MQR) com fator de esquecimento, conforme AGUIRRE (2000), LJUNG (1987), HEMERLY (1996), RÚBIO e SANCHÉZ (1996) e COELHO e COELHO (2004). Os algoritmos recursivos usam as estimativas do vetor $\theta(t)$, prevista a partir dos instantes anteriores ao instante de tempo $t$, para obtenção das estimativas $\theta(t+1)$, conforme indica a Equação (1).

$$
\hat{\theta}(t+1)=\hat{\theta}(t)+\text { atualização }
$$

Um modelo paramétrico quando utilizado para projeto de controladores não representa uma descrição complexa do sistema, mas deve descrever com grande precisão as características dinâmicas essenciais para o projeto do controlador. Diversos modelos paramétricos podem ser utilizados para representar os sistemas, entre eles, linear do tipo ARMAX, dado pela Equação (2).

$$
A\left(z^{-1}\right) y(t)=z^{-d} B\left(z^{-1}\right) u(t-1)+C\left(z^{-1}\right) e(t)
$$

onde:

$z^{-1}$ - operador atraso, tal que $z^{-1} y(t)=y(t-1)$;

$u(t)$ e $y(t)$ - são respectivamente o sinal de controle e a saída do sistema;

$A\left(z^{-1}\right)=1+a_{1} z^{-1}+a_{2} z^{-2}+\ldots+a_{n a} z^{-n a} ;$ denominador da função de transferência do sistema;

$B\left(z^{-1}\right)=b_{0}+b_{1} z^{-1}+b_{2} z^{-2}+\ldots+b_{n b} z^{-n b}$; numerador da função de transferência do sistema;

$C\left(z^{-1}\right)=1+c_{1} z^{-1}+c_{2} z^{-2}+\ldots+c_{n c} z^{-n c} ;$ numerador da função de transferência do filtro auto regressivo; 


\section{XIX \\ CENEMI}

$e(t)$ - ruído branco de média nula;

$d$ - atraso de transporte do sistema;

$n_{a}, n_{b}, n_{c}$ - ordem dos polinômios A, B e C.

Com $C\left(z^{-1}\right)=1$, o modelo ARMAX, resulta no modelo ARX, que pode ser escrito conforme o modelo de regressão linear mostrado na Equação (3).

$$
y(t)=\phi^{T}(t-1) \theta+e(t)
$$

onde:

$\phi^{T}(t-1)=\left[-y(t-1),-y(t-2), \ldots,-y\left(t-n_{a}\right), u(t-1), u(t-2), \ldots, u\left(t-1-n_{b}\right)\right]$

$\theta^{T}=\left[a_{1}, a_{2}, \ldots, a_{n_{a}}, b_{0}, b_{1}, \ldots, b_{n_{b}}\right]$

A estimação recursiva do vetor de parâmetros $\theta^{T}$ é regida pelas seguintes equações:

$$
\begin{gathered}
\theta(t)=\theta(t-1)+\mathrm{K}(t)\left[y(t)-\phi^{T}(t) \theta(t-1)\right] \\
\mathrm{K}(t)=P(t-1) \phi(t)\left[I-\phi^{T}(t) P(t-1) \theta(t)\right]^{-1} \\
P(t)=\frac{P(t-1)}{\lambda e}\left[I-\mathrm{K}(t) \phi^{T}(t)\right]
\end{gathered}
$$

onde :

$\mathrm{K}(t)$ - ganho do estimador;

$P(t)$ - matriz de covariancia dos parâmetros estimados;

$\lambda e$ - fator de esquecimento;

$I$ - matriz identidade.

No projeto do controlador via imposição de pólos, adota-se o modelo ARX, obtido a partir da Equação (3), e mostrado na Equação (7).

$$
y(t)=y_{u}(t)+n(t)=\frac{z^{-d} B\left(z^{-1}\right)}{A\left(z^{-1}\right)} u(t-1)+\frac{1}{A\left(z^{-1}\right)} e(t)
$$

onde:

$y_{u}(t)$ - modelo do sistema;

$n(t)$ - modelo da perturbação. 


\section{CONTROLADOR PD VIA IMPOSIÇÃO DE PÓLOS}

A Equação (8) mostra a representação da ação de controle do controlador PD no plano contínuo (OGATA 2010).

$$
G_{c}(s)=\frac{U(s)}{E(s)}=K_{p}\left(1+T_{d} s\right)
$$

onde, $K_{p}$ é o ganho proporcional e $T_{d}$ é tempo derivativo.

A forma discreta da Equação (8) é obtida a partir de uma aproximação de $s$ dada por QUILES et al. (2002), conforme Equação (9).

$$
s=\frac{1-z^{-1}}{T_{a}}
$$

onde, $T_{a}$ é o tempo de amostragem.

Substituindo o valor de $s$ da Equação (9) na Equação (8), tem-se como resultante a Equação (10) .

$$
G_{c}(z)=K_{p} \cdot\left(\frac{T_{a}+T_{d}}{T_{a}}\right)-K_{p} \cdot\left(\frac{T_{d} z^{-1}}{T_{a}}\right)=q_{0}+q_{1} z^{-1}=\frac{Q(z)}{P(z)}=\frac{q_{0} z+q_{1}}{z}
$$

A Equação (10) representa o controle PD na forma discretizada, e aplicando a transformada $Z$ inversa encontra-se a equação de diferenças que descreve o comportamento da variável de controle $u(k)$ do sistema.

$$
u(k)=q_{0} e(k)+q_{1} e(k-1)
$$

Os parâmetros $q_{0}$ e $q_{1}$ são determinados pela solução da equação característica do sistema com controlador em malha fechada.

O sistema em estudo é representado pela seguinte função de transferência discreta de segunda ordem.

$$
G_{p}(z)=\frac{B(z)}{A(z)}=\frac{b_{1} z+b_{2}}{z^{2}+a_{1} z+a_{2}}
$$

A função de transferência em malha fechada do conjunto controlador-planta é dada por:

$$
M(z)=\frac{G_{c}(z) \cdot G_{P}(z)}{1+G_{c}(z) \cdot G_{P}(z)}=\frac{B(z) \cdot Q(z)}{A(z) \cdot P(z)+B(z) \cdot Q(z)}
$$


O denominador da Equação (13) é o polinômio característico do controlador-planta, como mostra a Equação (14).

$$
P_{c}(z)=z^{3}+\left(a_{1}-1+b_{1} q_{0}\right) z^{2}+\left(a_{2}-a_{1}+b_{1} q_{1}+b_{2} q_{0}\right) z+b_{2} q_{1}-a_{2}
$$

Definindo $z_{1}=v+j \omega$ e $z_{2}=v-j \omega$ como sendo os pólos dominantes desejados para 0 sistema com controlador em malha fechada, tem-se o polinômio $P_{d}(z)=\left(z-z_{1}\right)\left(z-z_{2}\right)$ como uma parcela do polinômio característico desejado em malha fechada, representado pela Equação (15).

$$
P_{d}(z)=z^{2}-2 v z+v^{2}+\omega^{2}
$$

Como o polinômio característico da Equação (14) é de terceira ordem, e o produto dos pólos desejados é um polinômio de segunda ordem, um polinômio observador de primeira ordem; $P_{o}(z)=(z-p)$, é multiplicado pela Equação (15), resultando na Equação (16).

$$
P_{D}(z)=z^{3}-(2 v+p) z^{2}+\left(v^{2}+\omega^{2}+2 v p\right) z-v^{2} p-\omega^{2} p
$$

Igualando $P_{c}(z)$ à $P_{D}(z)$ os parâmetros $p, q_{0}$ e $q_{1}$ são obtidos conforme abaixo.

$$
\begin{gathered}
p=\frac{b_{1} b_{2}\left(a_{2}-\left(v^{2}+\omega^{2}\right)\right)-b_{2}{ }^{2}\left(a_{1}+2 v\right)}{b_{1}{ }^{2}\left(v^{2}+\omega^{2}\right)+2 b_{1} b_{2} v+b_{2}{ }^{2}} \\
q_{0}=-\frac{\left(a_{1}+2 v+p\right)}{b_{1}} \\
q_{1}=-\frac{\left(v^{2}+\omega^{2}\right) p}{b_{2}}
\end{gathered}
$$

Com o intuito de analisar o desempenho do robô sob ação do controlador PD, são definidas as seguintes especificações de desempenho: sobre-sinal máximo de $25 \%$ e erro em regime permanente de $\pm 2 \%$ do valor de referência.

Com as especificações de desempenho e o comportamento do polinômio característico definidos, os pólos dominantes discretos em malha fechada obtidos e ajustados para projeto dos controladores dos elos 1 e 2 são: $z_{1}=z_{2}=0,7 \pm 0,07 i$. 


\section{RESULTADOS OBTIDOS PARA OS ELOS DO ROBÔ}

As Figuras 3 e 4 mostram os sinais de entrada e saída dos elos 1 e 2 do robô manipulador, usados para o modelamento dos elos. Com o sistema em malha aberta, e utilizando o MQR considerando atraso de transporte $d=1$ para os dois elos, foram obtidos os modelos abaixo, na amostra 290.

$$
\begin{array}{r}
y_{1}(t)=0,9669 y_{1}(t-1)+0,0298 y_{1}(t-2)+0,0065 u_{1}(t-1)+0,0172 u_{1}(t-2) \\
y_{2}(t)=0,9674 y_{2}(t-1)+0,0288 y_{2}(t-2)+0,0051 u_{2}(t-1)+0,0406 u_{2}(t-2)
\end{array}
$$

As Figura 5 e 6 mostram as saídas reais e as saídas estimadas dos elos 1 e 2 do robô manipulador considerando todas amostras dos sinais reais.

As Figuras 7 e 8 mostram as saidas simuladas dos elos com os controladores PD adaptativos projetados. As respostas são em malhas fechadas, para referências em sequências de pulsos. Na determinação das saídas, os parâmetros dos controladores eram determinados para cada amostra assim como as variáveis de controle. Os modelos dos elos dados na Equação (20) e Equação (21), representaram o sistema real nesta fase de controle.

As Figuras 9 e 10 mostram as variáveis de controle dos elos 1 e 2 .

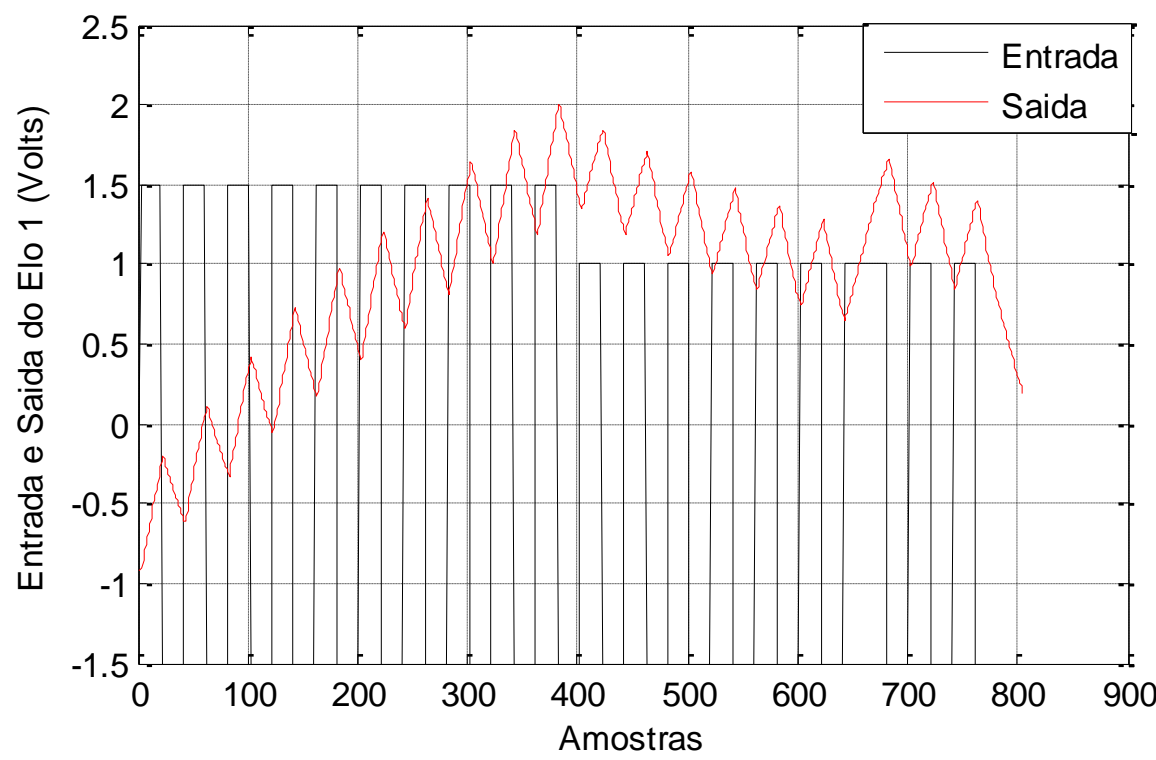

Figura 3. Sinais de entrada e saída do elo 1 do robô. 


\section{XIX \\ CENEMI}

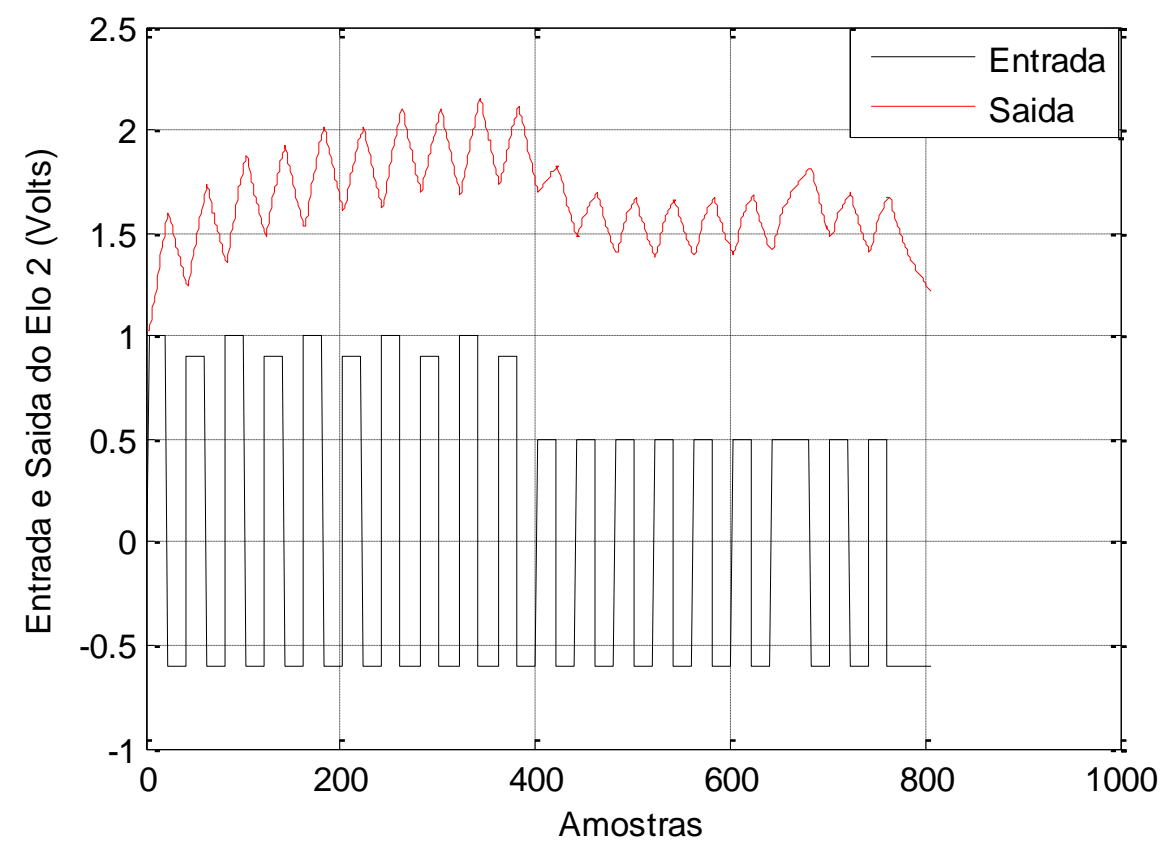

Figura 4. Sinais de entrada e saída do elo 2 do robô.

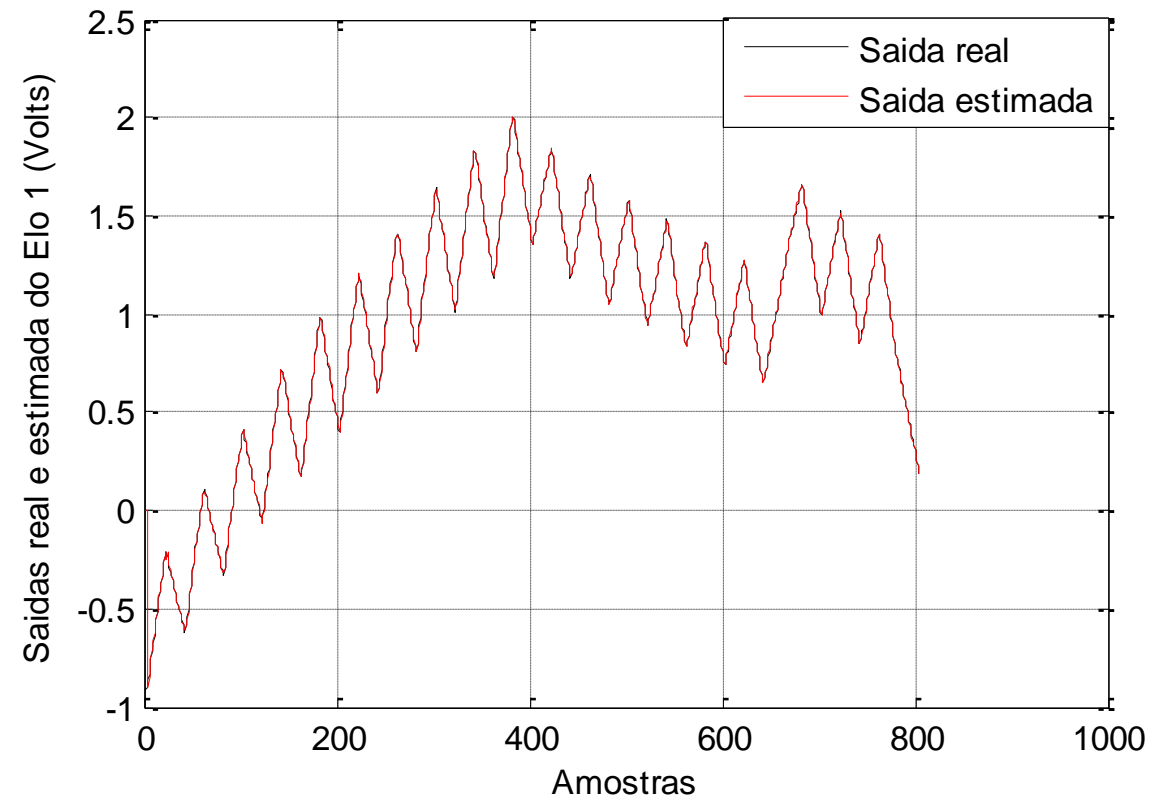

Figura 5. Saídas real e estimada do elo 1 do robô. 


\section{XIX \\ CENEMI}

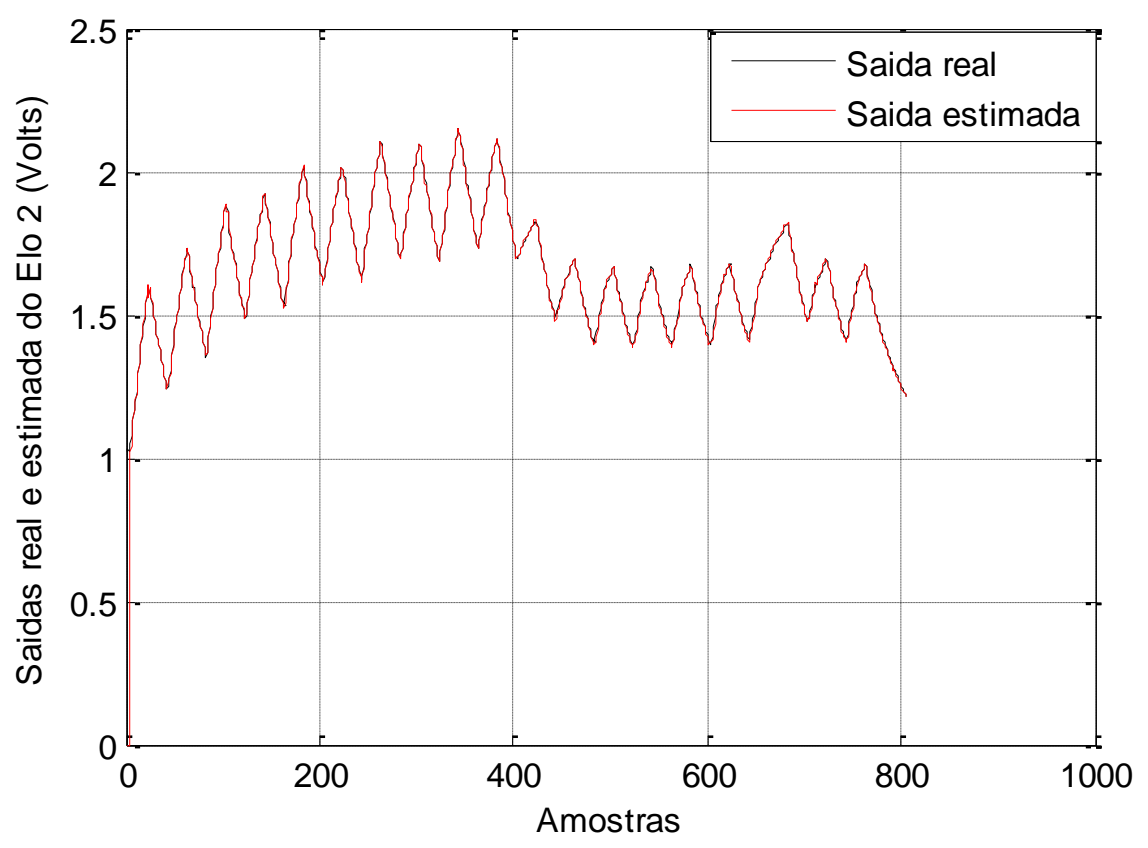

Figura 6. Saídas real e estimada do elo 2 do robô.

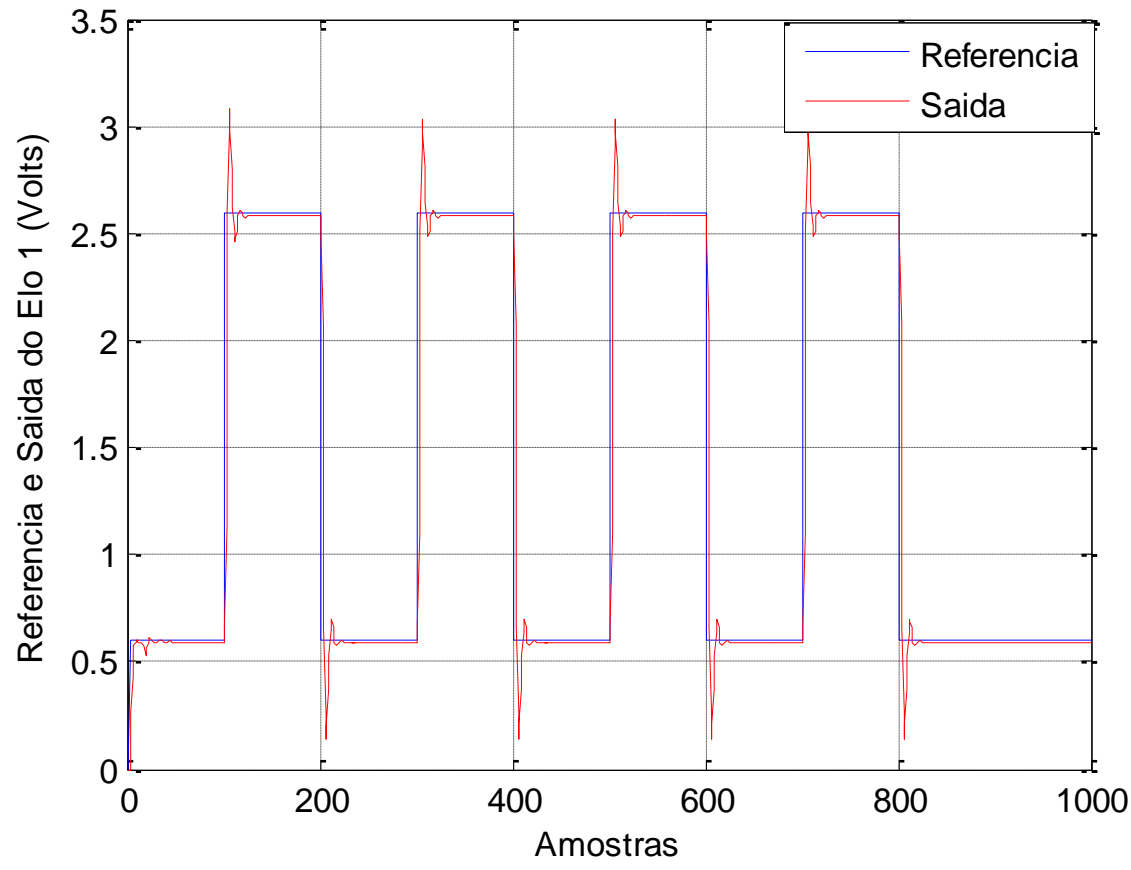

Figura 7. Saída simulada do elo 1 do robô sob ação do controlador. 


\section{XIX \\ CENEMI}

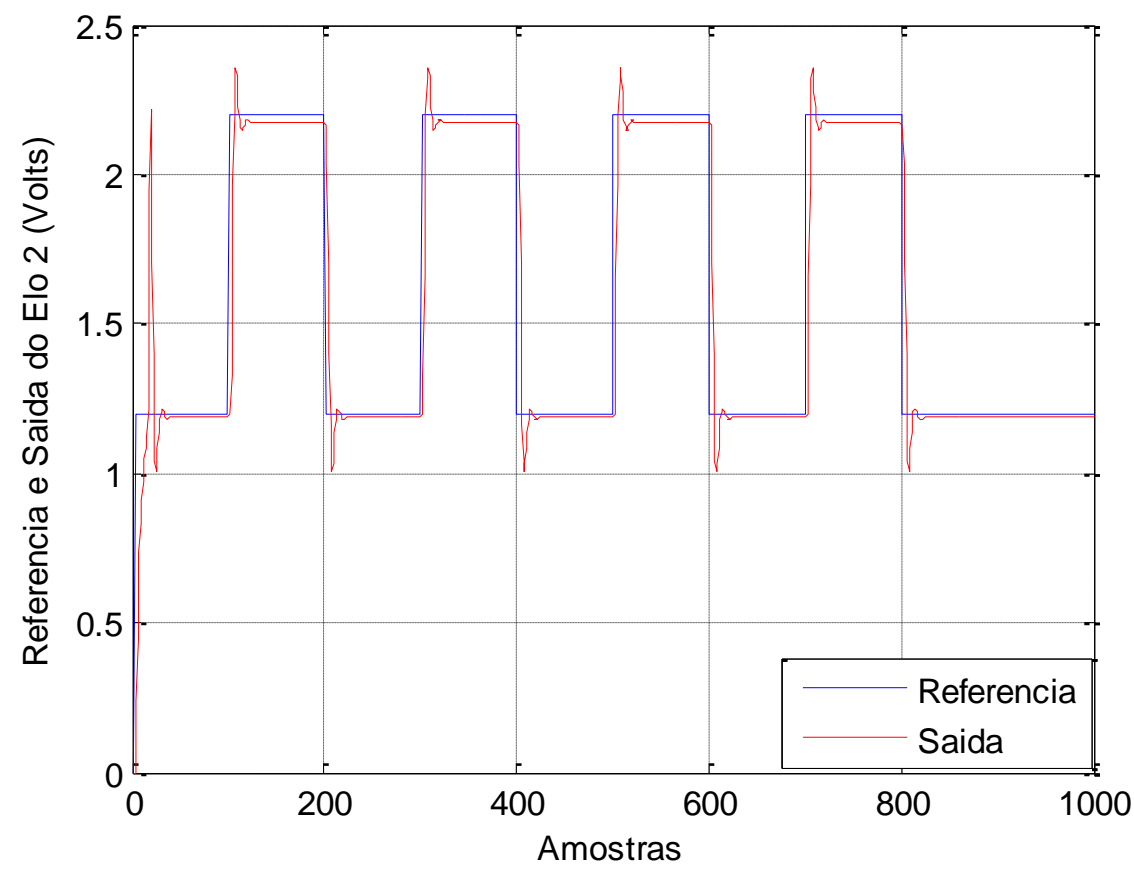

Figura 8. Saída simulada do elo 2 do robô sob ação do controlador.

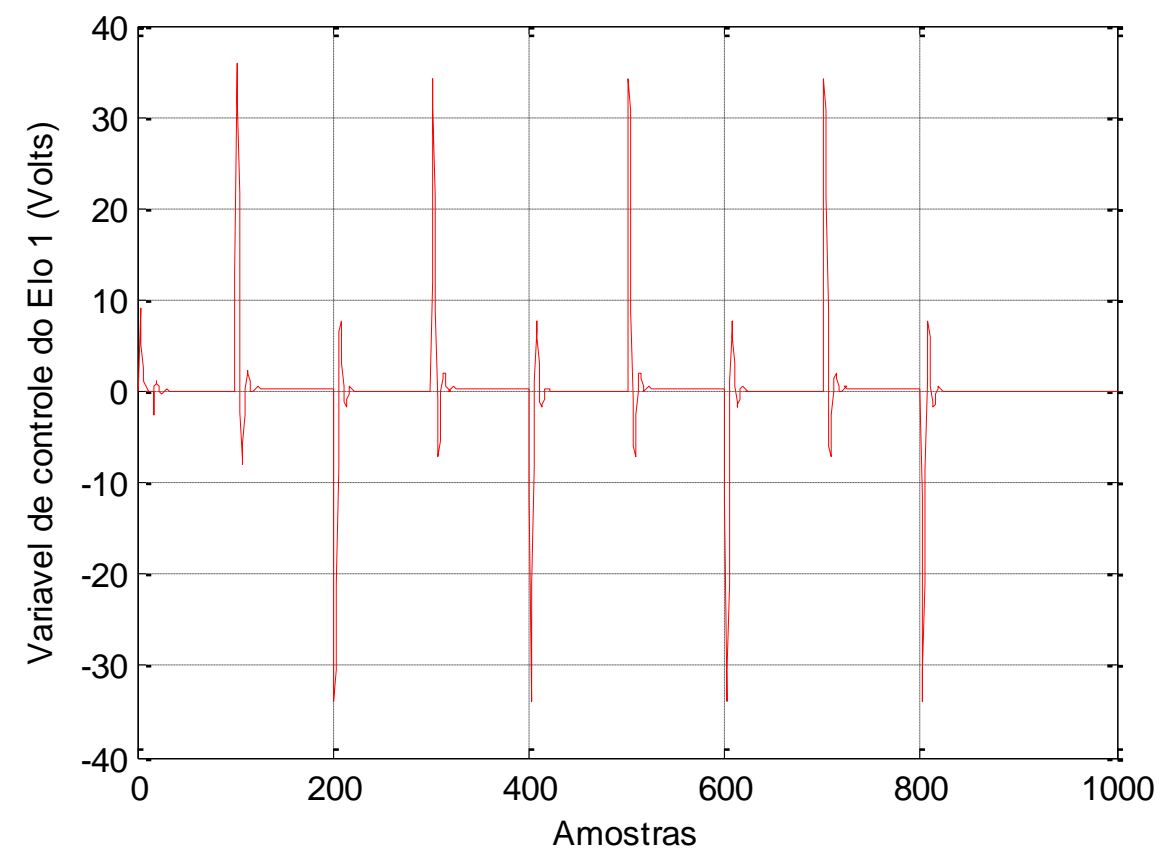

Figura 9. Variavel de controle do elo 1 do robô. 


\section{XIX \\ CONEMI}

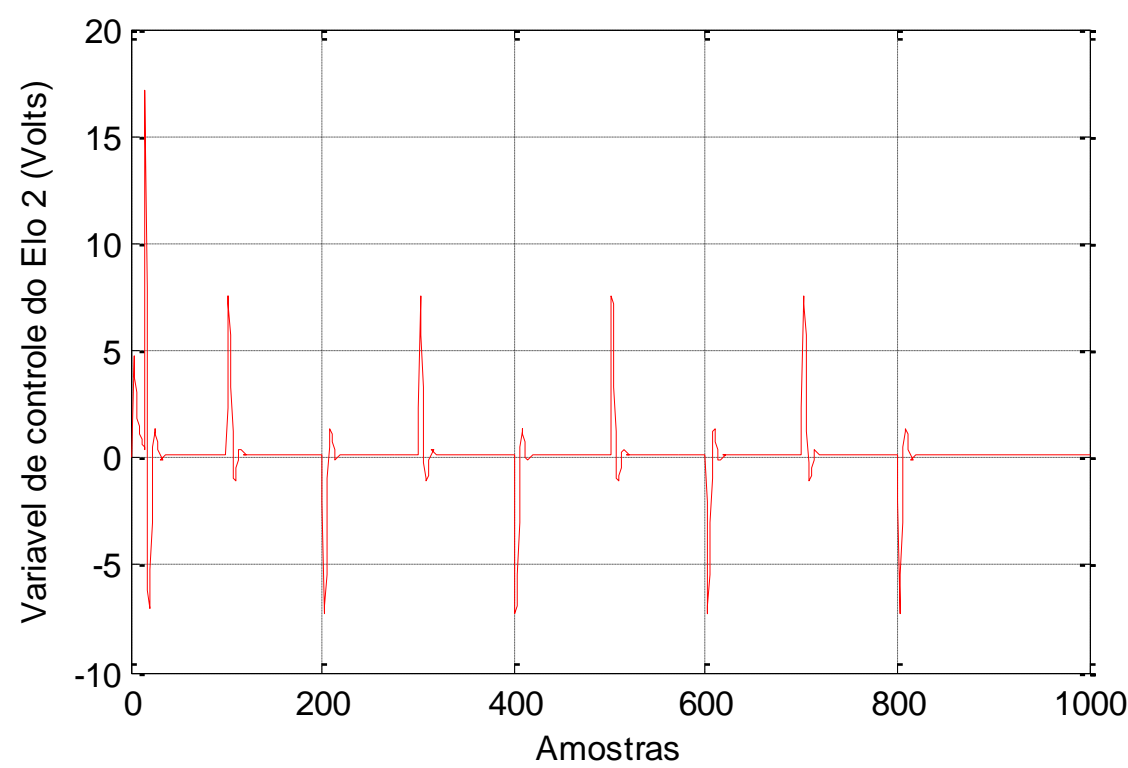

Figura 10. Variavel de controle do elo 2 do robô.

\section{CONCLUSÃO}

Este trabalho apresentou a técnica de identificação linear e o projeto e implementação de controladores PD adaptativos, para dois elos de um robô manipulador de cinco graus de liberdade. A identificação dos modelos foi realizada utilizando-se o algoritmo MQR, considerando a dinâmica dos dois elos do robô acoplada.

Os projetos dos controladores PD adaptativos foram realizados através de simulação, e usando os modelos obtidos para os elos. A Erro! Fonte de referência não encontrada. mostrou que a saída do sistema sob ação do controlador STR acompanhou adequadamente a referência estabelecida para o elo 1 com sobre-sinal de $25 \%$ e erro em regime dentro do critério de $2 \%$. A Erro! Fonte de referência não encontrada. mostrou que a saída do sistema sob ação do controlador STR acompanhou adequadamente a referência estabelecida para o elo 2 com sobre-sinal de $20 \%$ e erro em regime dentro do critério de $2 \%$.

\section{REFERÊNCIAS}

AGUIRRE, L. A. Introdução à Identificação de Sistemas: Técnicas lineares e não-lineares aplicadas a sistemas reais. 2a edição, Belo Horizonte, MG, UFMG, 2000.

BEHAR, A. A. e IRANZO, M.M. Identificacíon y Control Adaptativo. Ed. Prentice Hall 1a edição. Madri, 2003.

Coelho, A.A.R., Coelho, L.S. Identificação de Sistemas Dinâmicos Lineares. 1a ed., Florianópolis: Ed. Universidade Federal de Santa Catarina, 2004. 


\section{$\mathbf{X I X}$ \\ CENEMI}

CRAIG, J. J. Introduction to Robotics: Mechanics and Control. Addison-Wesley, 2a ed., 1989.

HEMERLY, E. M. Controle Por Computador de Sistemas Dinâmicos. Ed. Edgard Blucher, LTDA, S. Paulo, Brasil, 1996.

KOIVO, A. J., GUO, T. Adaptive Linear Controller for Robotic Manipulator. IEEE Transactions on Automatic Control, v. AC-28, pp. 162-171, 1983.

LJUNG, L. System Identification: Theory for the User. Ed. Prentice - Hall Inc, Englewood Cliffs, New Jersey, 1987.

OGATA, K. Engenharia de Controle Moderno. $5^{\text {a }}$ ed., São Paulo - SP, Ed. Pearson, 2010.

QUILES, E., MORANT, F., GARCIA, E., BLASCO, R., CORRECHER, A. Control Adaptativo de um Sistema de Control Neumático. In: Conferência Iberoamericana em Sistemas Cibernética e Informática, Orlando, USA, 2002.

RÚBIO, F.R \& SÁNCHEZ, M.J.L. Control Adaptativo y Robusto. Secretariado de Publicaciones de la Universidad de Sevilla, Espanha, 1996.

SPONG, M. W., VIDYASAGAR, M. Robot Dynamics and Control.John Wiley \& Sons, 1989.

SHIH, M. C., TSENG, S. I. Identification and Position Control of a Servo Pneumático Cylinder. Control Engineering Practice, v. 3, n. 9, pp. 1285-1290, 1995. 